Methods We compared the accuracy of a handheld spirometer (COPD6, Vitalograph, UK) with a standard laboratory based spirometer (Spirostik, Geratherm, Germany) in 41 consecutive adult CF patients (mean age 39 years [SD 11.4], mean predicted FEV1 $60 \%$ [23], 16 male) attending our regional CF centre. All were experienced at performing spirometry. They were randomly assigned to either device and carried out 3 manoeuvres before repeating the test session the alternative spirometer. Both testing sessions were supervised and performed in the same clinic room. Patient coaching and feedback was witheld from the handheld spirometry session. Intra-session analysis of flow volume tracings was carried out for lab based testing sessions.

Results Acceptable paired results were obtained in 36 patients (88\%). The mean Spirostik FEV1 was $2.14 \mathrm{~L}$ (variability 0.06 [2.3\%]) and COPD6 2.02L (variability 0.09 [4.4\%]); mean difference between devices $0.12 \mathrm{~L}(5.6 \%)$. Eight of 36 (22\%) results were lower on the handheld device.

Analysis of the flow volume tracing was required for 6 patients $(16 \%)$ to either improve technique (3), or QC/eliminate artefact (3): where artefact was not eliminated, FEV1 was overestimated by 0.08 to $0.09 \mathrm{~L}(\sim 4.5 \%)$.

Conclusions The handheld device was easy to operate and accuracy and repeatability was considered to be acceptable for a telemedicine/home use application. However, differences between traditional clinic/laboratory spirometry results and telemedicine spirometry results were observed. When comparing spirometry results clinicians should have an appreciation of expected differences. Additional variables may exist where patients are unsupervised in a home setting.

\section{P255 REPRODUCIBILITY OF LUNG CLEARANCE INDEX (LCI) IN CLINICALLY STABLE ADULTS WITH MILD CYSTIC FIBROSIS (CF)}

${ }^{1}$ AR Horsley, ${ }^{1}$ A Shawcross, ${ }^{2} \mathrm{M}$ Oladapo, ${ }^{2} \mathrm{~A}$ Maitra, ${ }^{3} \mathrm{~S}$ Cunningham, ${ }^{4} \mathrm{AM}$ Jones, ${ }^{1} \mathrm{~S}$ Smith, ${ }^{5} \mathrm{~F}$ Gilchrist. 'University of Manchester, Manchester, UK; ${ }^{2}$ Royal Manchester Children's Hospital, Manchester, UK; ${ }^{3}$ Royal Hospital for Sick Children, Edinburgh, UK; ${ }^{4}$ Manchester Adult Cystic Fibrosis Centre, Manchester, UK; ${ }^{5}$ Royal Stoke University Hospital, Stoke, UK

10.1136/thoraxjnl-2016-209333.398

Background In order for lung clearance index (LCI) to be a clinically useful measurement, a better understanding is required of short-term variability. LCI-SEARCH is a longitudinal study in children and adults with CF, with LCI measured at each clinical review using a portable closed-circuit wash-in system (www.lcisearch.com). Here we report initial LCI repeatability from the adult cohort.

Methods LCI measurements were performed in triplicate using a closed-circuit wash-in method (Horsley et al. ERJ open). The most recent paired LCI measurements were included providing they were within 6 months of each other, the patient was deemed clinically stable by a physician and the patient scored $<2$ on a 4 point respiratory symptom score. Repeatability was assessed by Bland-Altman analysis.

Results Of 40 CF adults, paired data were available on 21 (7 subjects had completed only 1 assessment, 1 withdrawn, 11 clinically unstable). These 21 subjects (14 male) completed a median of 5 LCI measurements each (range 2-11), a median of 84 (range 42189) days apart. Mean age was 28 yrs, mean FEV1 $82 \%$ predicted, 11 pancreatic sufficient, 11 had never had pseudomonas infection.

Mean (SD) LCI at visit 1 was 8.68 (2.96) vs 8.73 (2.81) at visit $2(\mathrm{p}=\mathrm{ns})$. Median coefficient of variation for LCI was $3.9 \%$ (visit 1) and 4.2\% (visit 2). Mean change in LCI between visits was 0.05 (1\% of baseline LCI). 95\% limits of agreement (LOA) were $-1.1(-13.7) \%$ to $1.0(11.6) \%$ of baseline LCI. In this very mild cohort, 7 patients had normal LCI $(<7)$; exclusion of these did not substantially alter LOA $(-13.9$ to $13.1 \%)$. There was greater variability in FRC: mean bias $-1.5 \%$ of baseline (LOA 30 to $-33 \%)$.

Conclusions Even in this very mild cohort of CF adults, patients are frequently unwell or more symptomatic at routine review. Within-visit repeatability was good, and similar to previous reports. When clinically stable, LCI variability over a period of up to 6 months was approximately $\pm 10 \%$. Addition of more adult as well as paediatric data to this assessment will widen the applicability of these confidence intervals.

\section{P256 RESPIRATORY MUSCLE STRENGTH MEASUREMENTS IN PRIMARY SCHOOL CHILDREN}

NTS Gharbawi, EA Gaillard, M Viskaduraki, CS Beardsmore. University of Leicester, Leicester, UK

\subsection{6/thoraxjnl-2016-209333.399}

Background Previous tests of respiratory muscle strength have rarely included measurements of inspiratory pressure. As part of a study looking at ethnic differences in respiratory muscle strength, we have measured maximum inspiratory and expiratory pressures in primary school children.

Aim We sought to determine the success rate and within-test repeatability of respiratory muscle strength measurements.

Methods We measured spirometry, height and weight and respiratory muscle strength by measuring maximal inspiratory and expiratory pressure (MIP and MEP) using Carefusion Vyntus in children aged 5-11 yr. Children breathed through a mouthpiece and pneumotachograph attached to a shutter, while wearing a noseclip. After a period of tidal breathing the child breathed in to total lung capacity and then tried to exhale forcibly against the shutter. We measured maximal (peak) expiratory pressure (MEP). For measurements of MIP, the child exhaled towards residual volume before making an inspiratory effort against the occlusion. Manoeuvres were excluded if the peak pressures were less than $3.50 \mathrm{kPa}$. We reported the largest pressures recorded, provided that the second-best was no more than $20 \%$ below the best. We calculated the percent difference between best and 2nd best manoeuvres and compared mean percentage differences in MIP and MEP.

Results Two hundred and thirty-one children were studied. We obtained MEP on 199 and MIP on 216, and paired data for MIP and MEP on 165 (87 boys and 78 girls). Overall, MIP was higher than MEP (mean (SD) MIP $=7.26$ (1.92) $\mathrm{kPa}, \mathrm{MEP}=6.64$ (1.76) $\mathrm{kPa}, \mathrm{p}=0.002$ ). However, MEP tended to be bigger than MIP when the values were smaller (in the younger, smaller children) (Figure). There was no significant difference between\%difference MIP and\%difference MEP (mean (SD) $5.50 \pm 4.29$ and $4.68 \pm 3.96 \mathrm{kPa}$ respectively, $\mathrm{p}=0.07)$.

Conclusion The success rates of MIP and MEP measurements were $94 \%$ and $86 \%$ respectively, suggesting that MIP was easier for the children to perform. The success rate for paired measurements was $71 \%$. The repeatability of inspiratory and expiratory pressures was not different. We speculate that the change with age between which measurement was greatest (MIP or MEP) may reflect dysanaptic muscle development. 


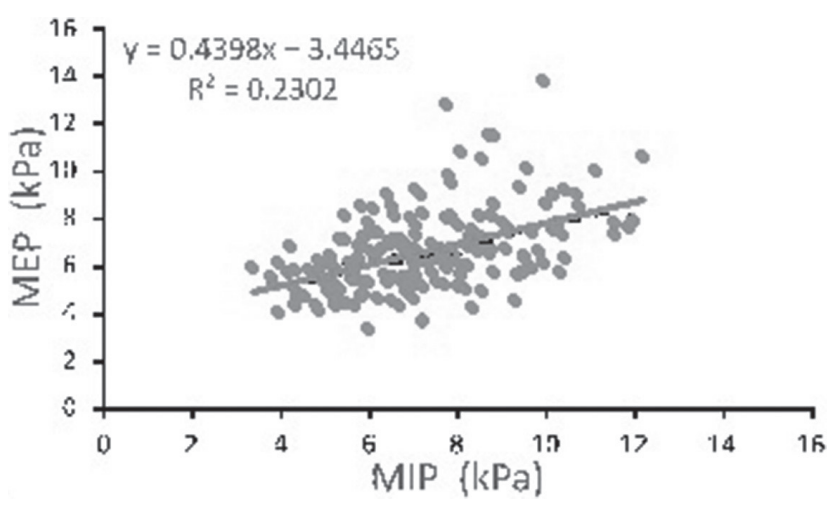

Abstract P256 Figure 1 MIP vs MEP (regression analysis $p=0.004$ )

\section{P257 UNDERSTANDING THE EFFECTS ON LUNG FUNCTION OF CHEST BINDER USE IN THE TRANSGENDER POPULATION}

${ }^{1} \mathrm{RJM}$ Cumming, ${ }^{2} \mathrm{~K}$ Sylvester, ${ }^{2} \mathrm{~F}$ Fuld. 'University of Cambridge, Cambridge, UK; ${ }^{2}$ Addenbrookes Hospital, Cambridge, UK

\subsection{6/thoraxjnl-2016-209333.400}

Introduction Chest binders are garments used for compression of breast tissue by transgender individuals. Deleterious consequences of binder reported include shortness of breath with associated reduced exercise tolerance and speech difficulties; some have suggested lung function is monitored in users of chest binders. ${ }^{1} \mathrm{We}$ conducted a study to investigate any respiratory deficits caused by chest binders as currently used in the transgender population. Methods We recruited 20 participants from the transgender community. All were assigned female at birth. Ages ranged from 1947 with median age 22; 4 were current smokers and 4 had mild to moderate asthma. All were habitual users of chest binders. Participants underwent spirometry testing and measures of chest circumference and posture with and without their own binder. The order of testing with or without the binder was random. Ethics approval was granted by the University of Cambridge.

Results Table 1 shows abnormal baseline lung function. The median FEV1/FVC is abnormally high but not acutely influenced by the binder. The standard residual of all forced spirometric values was significantly $(\mathrm{p}<0.001)$ below predicted values (based on sex assigned at birth); peak expiratory flow (PEF) values were also lower than predicted. There was a significant reduction in expiratory vital capacities, both SVC and FVC $(p<0.01)$ when the binder was on but no other significant acute change. On average chest circumference was reduced by the binder. There was no average change in thoracic kyphosis due to high variability.

Conclusions Transgender individuals using chest binders have abnormal lung function. The acute effect of wearing the binder appears to be an overall volume reduction with little other change. Abnormal lung function in the population may indicate a chronic effect of binder usage or generally poor respiratory health. However, due to the small size and timeframe of the study no control population was tested and thus a systematic error cannot be ruled out.
Abstract P257 Table 1 Median spirometry values acquired with the binder off vs. on. A reduction in vital capacity is seen with the binder on.

\begin{tabular}{|c|c|c|c|c|c|c|c|c|c|c|}
\hline & \multicolumn{10}{|c|}{ Summary of spirometry values } \\
\hline & \multicolumn{2}{|l|}{ FVC } & \multicolumn{2}{|l|}{ FEV1 } & \multicolumn{2}{|c|}{ FEV1/FVC } & \multicolumn{2}{|l|}{ PEF } & \multicolumn{2}{|l|}{ SVC } \\
\hline & Off & On & Off & On & Off & On & Off & On & Off & On \\
\hline $\begin{array}{l}\text { Median } \\
\text { (litres) }\end{array}$ & 4.35 & 4.29 & 3.46 & 3.53 & 0.82 & 0.82 & 394.2 & 397.2 & 4.22 & 4.12 \\
\hline $\begin{array}{l}\text { Median } \\
\text { (SR) }\end{array}$ & -0.54 & -0.65 & -0.92 & -1.30 & -0.62 & -0.46 & & & & \\
\hline $\begin{array}{l}\text { Median } \\
\text { (\% Pred.) }\end{array}$ & & & & & & & 87.7 & 87.0 & & \\
\hline $\begin{array}{l}p \text { value } \\
\text { off vs on }\end{array}$ & 0.0062 & & 0.0701 & & 0.3603 & & 0.2349 & & 0.0008 & \\
\hline
\end{tabular}

\section{REFERENCE}

1 Davies S, Papp VG, Antoni C. Voice and communication change for gender nonconforming individuals: Giving voice to the person inside. Int J Transgenderism 2015;16:117-159.

\section{P258 INFANT LUNG FUNCTION TESTING: A NEW APPROACH USING A RAPID, PORTABLE SYSTEM FOR MEASURING LUNG CLEARANCE INDEX (LCI) IN HEALTH AND DISEASE}

${ }^{1}$ A Shawcross, ${ }^{1} \mathrm{CS}$ Murray, ${ }^{2} \mathrm{~J}$ Kirkby, ${ }^{3} \mathrm{~J}$ Miles, ${ }^{2} \mathrm{~K}$ Pike, ${ }^{3} \mathrm{~S}$ Rees, ${ }^{2} \mathrm{P}$ Aurora, ${ }^{1} \mathrm{~A}$ Horsley. 'University of Manchester, Centre for Respiratory Medicine and Allergy, Manchester, UK; ${ }^{2}$ Respiratory, Critical Care and Anaesthesia Section, Institute of Child Health, UCL, London, UK; ${ }^{3}$ Lung Function Unit, Great Ormond Street Hospital for Children, London, UK

\subsection{6/thoraxjnl-2016-209333.401}

Introduction Lung clearance index (LCI) is a sensitive measure of lung disease in infants, with potential applications in clinical practice and research. However, measuring LCI in infants is technically challenging and there is no simple method of assessing LCI outside of specialist research laboratories in this population.

We have previously described an alternative method of measuring LCI, in which expired gas is collected and analysed to derive functional residual capacity (FRC) and LCI without directly measuring flow. This eliminates one of the major technical challenges, whilst also reducing the system's dead space. This method is highly accurate in vitro, with a mean accuracy of FRC measurement to within $1 \%$, down to FRC of $100 \mathrm{ml} .{ }^{1}$ The method does not require large external gas tanks, and washout is performed breathing room air, making the system fully portable.

Aim To assess the performance of this method in vivo.

Method Healthy controls and infants with CF are currently being recruited to undergo LCI measurement using this method. Practical applicability of the system is determined by the number of successful tests and within-subject repeatability, defined as coefficient of variation (CV\%) of same-visit repeats. Comparison will be made with LCI measurements obtained using a respiratory mass spectrometer, currently considered the gold standard for infant LCI measurement. 